\title{
Fluctuations and Transport in a Stirred Fluid with a Mean Gradient
}

\author{
J. P. Gollub, (1),(2),(3),(a) J. Clarke, ${ }^{(2)}$ M. Gharib, ${ }^{(2)}$ B. Lane, (1) and O. N. Mesquita (1),(b) \\ (1) Physics Department, Haverford College, Haverford, Pennsylvania 19041 \\ ${ }^{(2)}$ Institute for Nonlinear Science, The University of California at San Diego, La Jolla, California 90237 \\ ${ }^{(3)}$ Physics Department, The University of Pennsylvania, Philadelphia, Pennsylvania 19104
}

(Received 18 September 1991)

The effective thermal diffusivity $D^{*}$ and the probability distribution of temperature fluctuations are measured in a stirred fluid across which a temperature gradient is maintained. A distinct mixing transition is observed for $D^{*}$ as a function of Reynolds number $R$. Above the transition, the distribution is strongly non-Gaussian and approaches an exponential $\exp (-|\delta T| / \beta \xi)$, where $\beta$ is the local temperature gradient and $\xi$ the correlation length.

PACS numbers: $47.25 . J n, 05.40 .+\mathrm{j}$

Enhanced transport properties are among the basic characteristics of turbulent flows. Several recent experiments have provided detailed measurements for the transport of heat and angular momentum in situations where these quantities are dynamically active, for example, in thermal convection [1] and Taylor-Couette flow [2]. It is equally interesting to consider the passive transport of scalar quantities such as impurity concentration or heat in the presence of gradients of these quantities. The concept of an effective or eddy diffusivity is often employed to describe the enhancement. Its value is roughly of the order of $\xi_{V} V$, where $\xi_{V}$ is a typical eddy size or velocity correlation length, and $V$ is the rms velocity. This quantity can be many orders of magnitude larger than the molecular diffusivity for material or thermal energy. The subject of stirring and mixing in fluids that are not necessarily turbulent has had a renaissance due in part to an appreciation of its conceptual connection to chaotic dynamics [3].

The fluctuations of the local temperature or concentration contain considerable information about both dynamics and transport processes. Fluctuations of local velocity differences in turbulent flows are known to be nonGaussian, a reflection of the phenomenon of intermittency [4]. Recently Pumir, Shraiman, and Siggia [5] proposed a simplified model of the random advection of a passive scalar in a closed volume across which a steady gradient $\beta$ is maintained, and which is stirred with a velocity correlation length $\xi_{V}$ much less than the size $L$ of the system. Their model, which was stimulated by earlier observations [1] of exponential probability distributions in the case of active thermal convection, leads to the prediction that exponential tails will occur in the probability distribution function (PDF) of the passive scalar as well, rather than the Gaussians that might have been expected for that case. The extended tails, with an expected decay constant of about $\left(\beta \xi_{V}\right)^{-1}$, arise from the fact that fluid at a given location comes from other locations where the local mean value is systematically larger or smaller. Gaussian distributions are expected (based on numerical simulations) for weaker mixing, and complex nonmonotonic distributions are predicted when the parameter
$\xi_{V} / L$ is not small, so that fluid from the boundaries reaches the measurement location with little mixing. The model indicates that it is not necessary to look at gradients or differences to detect non-Gaussian behavior, and that strong turbulence is not required.

The model of Ref. [5] is a phenomenological model of mixing and is not systematically derived from the hydrodynamic equations. However, the prediction of exponential tails for passive scalar transport is supported by earlier [6] and recent [7] numerical simulations. The problem of predicting passive transport coefficients and statistics of fluctuations is surprisingly complicated mathematically, because the large scales relevant to bulk transport are affected by a wide range of smaller scales, as has been pointed out in theoretical studies of model systems by Avellaneda and Majda [8]. Other approaches to passive scalars have been discussed [9], and mechanisms different from that of Ref. [5] could be relevant [10]. Thus, it is desirable to determine experimentally whether and when exponential tails actually occur for passive transport.

In this paper, we report experimental studies of both the effective thermal diffusivity and the statistical fluctuations of the temperature field in a stirred fluid with a steady (but small) temperature gradient. The system (Fig. 1) is a closed box $7.5 \mathrm{~cm}$ deep constructed of Plexiglas, with horizontal interior dimensions $L=12.5 \mathrm{~cm}$ (parallel to the temperature gradient) and $25 \mathrm{~cm}$. The fluid (water and water-glycerol mixtures) is mixed by a rigid oscillating grid formed by a hexagonal array of holes (diameter $d=0.48 \mathrm{~cm}$ ) in a sheet of Plexiglas 0.3 $\mathrm{cm}$ thick. If we take $d$ as an estimate of the characteris-

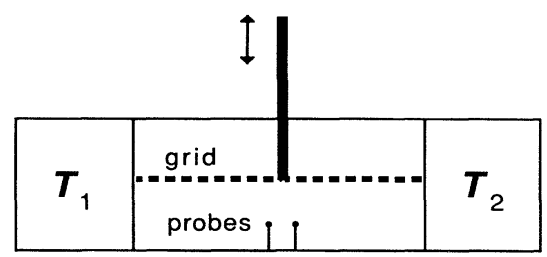

FIG. 1. Sketch of the apparatus showing the oscillating grid and temperature-control baths. 
tic scale $\xi_{V}$ of the "random" part of the velocity field, then the parameter $\left(\xi_{V} / L\right)^{2}$ is 0.037 . The grid has negligible thermal diffusivity compared to that of the stirred fluid, and oscillates with periods $0.5-10 \mathrm{~s}$ through the center $40 \%$ of the fluid depth.

Copper plates $0.3 \mathrm{~cm}$ thick separate the active fluid from adjacent temperature-controlled thermal baths. The applied temperature difference across the entire cell is typically $3 \mathrm{~K}$, a value sufficiently small that induced thermal convection is insignificant in comparison to the flows produced by active stirring. Temperature stability better than $0.01 \mathrm{~K}$ even in the presence of a large heat flux is typically obtained, and is necessary because the gradients in the interior are small, typically $0.03-0.1$ $\mathrm{K} / \mathrm{cm}$.

Local temperatures and temperature differences are measured by small thermistor probes of diameter 0.05 $\mathrm{cm}$, whose frequency response is estimated to be about 50 $\mathrm{Hz}$. This bandwidth is more than sufficient for the flows considered. Measurements are made along a line parallel to the temperature gradient and typically $5 \mathrm{~mm}$ below the lowest point of the oscillating grid.

We base the Reynolds number $R$ on the grid hole diameter $d$ and the maximum velocity of the fluid through the holes. The range $100<R<6000$ has been explored. Visualization using anisotropic particles indicates that for $R>1000$ the turbulence is fairly well developed, with scales considerably smaller than $d$ being excited. The turbulent fluctuations fill the cell, though they are somewhat attenuated near the top and bottom. An impurity introduction near one wall of the cell soon forms a front that broadens gradually, with times of the order of $100 \mathrm{~s}$ being required to mix uniformly across the cell. Some velocity measurements are obtained by laser Doppler velocimetry. While the maximum vertical velocity can be as large as $50 \mathrm{~cm} / \mathrm{s}$ at $R=5000$, the rms horizontal velocity is much smaller, about $V=1.5 \mathrm{~cm} / \mathrm{s}$.

We begin by describing the behavior of the effective thermal diffusivity $D^{*}=K^{*} / \rho c$, where the effective

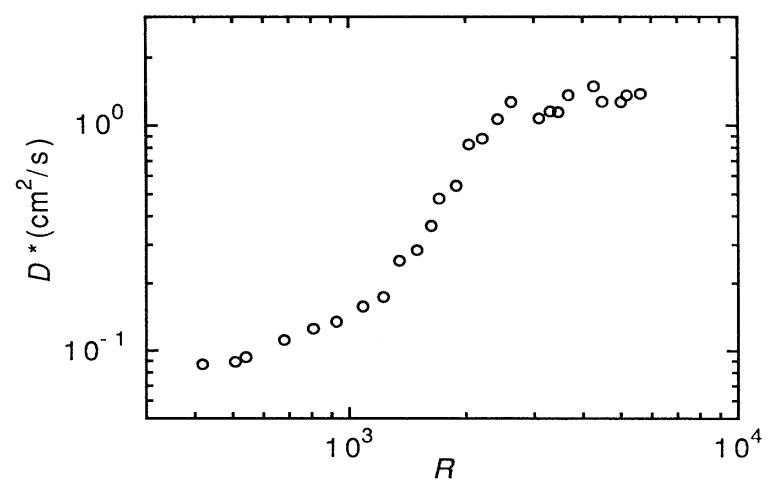

FIG. 2. Effective thermal diffusivity $D^{*}$ as a function of Reynolds number $R$, showing a rapid rise or "mixing transition" as the turbulent cascade develops, and a slow growth for higher $R$. thermal conductivity $K^{*}$ is obtained from the ratio of the total measured heat flux $F$ to the local temperature gradient, $\rho$ is the density, and $c$ is the heat capacity. This quantity is shown as a function of $R$ in Fig. 2. It reveals a gradual rise at "low" $R$ and a rapid rise starting near $R=10^{3}$; this is a reflection of the corresponding behavior of $\nabla T$, which declines rapidly over the same range. For water at $R=5000$, the enhancement factor of the thermal diffusivity is approximately $10^{3}$. Typical values of $D^{*}$ above the mixing transition are consistent with $V d$. For higher $R, D^{*}$ continues to rise, perhaps linearly, but the accuracy and the range in $R$ of our present experiments are not sufficient to define this dependence precisely.

A nondimensional heat flux or Nusselt number $\mathrm{Nu}$ $=F / F_{\text {cond }}$ may be defined by the ratio of the turbulent and conductive fluxes. This quantity shows a much simpler smooth power-law increase that is well approximated by the expression $N=0.32 R^{0.64} \pm 0.05$ over the entire range $500<R<6000$. However, it is primarily determined by the thickness of the boundary layers at the hot and cold surfaces, and is not a bulk property.

The temperature fluctuations are usefully characterized by the spatial correlation function $C_{T}(r)$ $=\langle\delta T(r) \delta T(0)\rangle /\left\langle(\delta T)^{2}\right\rangle$, which is shown in Fig. 3. It is well approximated as an exponential with a correlation length $\xi_{T}=8 \mathrm{~mm}$ at $R=3700$. We are not able to measure $\xi_{V}$ directly, though we estimate it to be of the order of the grid hole diameter $d$. Thus, the correlation lengths for velocity and temperature are comparable.

Typical probability distributions for temperature fluctuations are shown in Fig. 4 for $R=600$ (below the mixing transition) and $R=3700$ (above the mixing transition). The fluctuations are nondimensionalized by the standard deviation $\sigma$ (even when the distributions are not Gaussian). The distribution shown in Fig. 4(a) is adequately fitted by a Gaussian, while that in 4(b) has welldefined tails that are fitted much better by exponentials. In fact, the observed fluctuations at $\delta T / \sigma=4$ are more probable than those predicted by a Gaussian with the

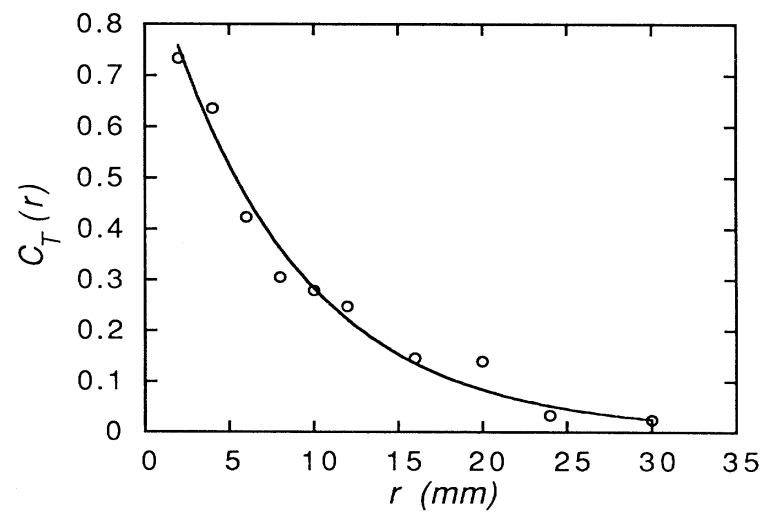

FIG. 3. Spatial correlation function $C_{T}(r)$ of the temperature field, showing roughly exponential decay in the turbulent regime at $R=3700$. 

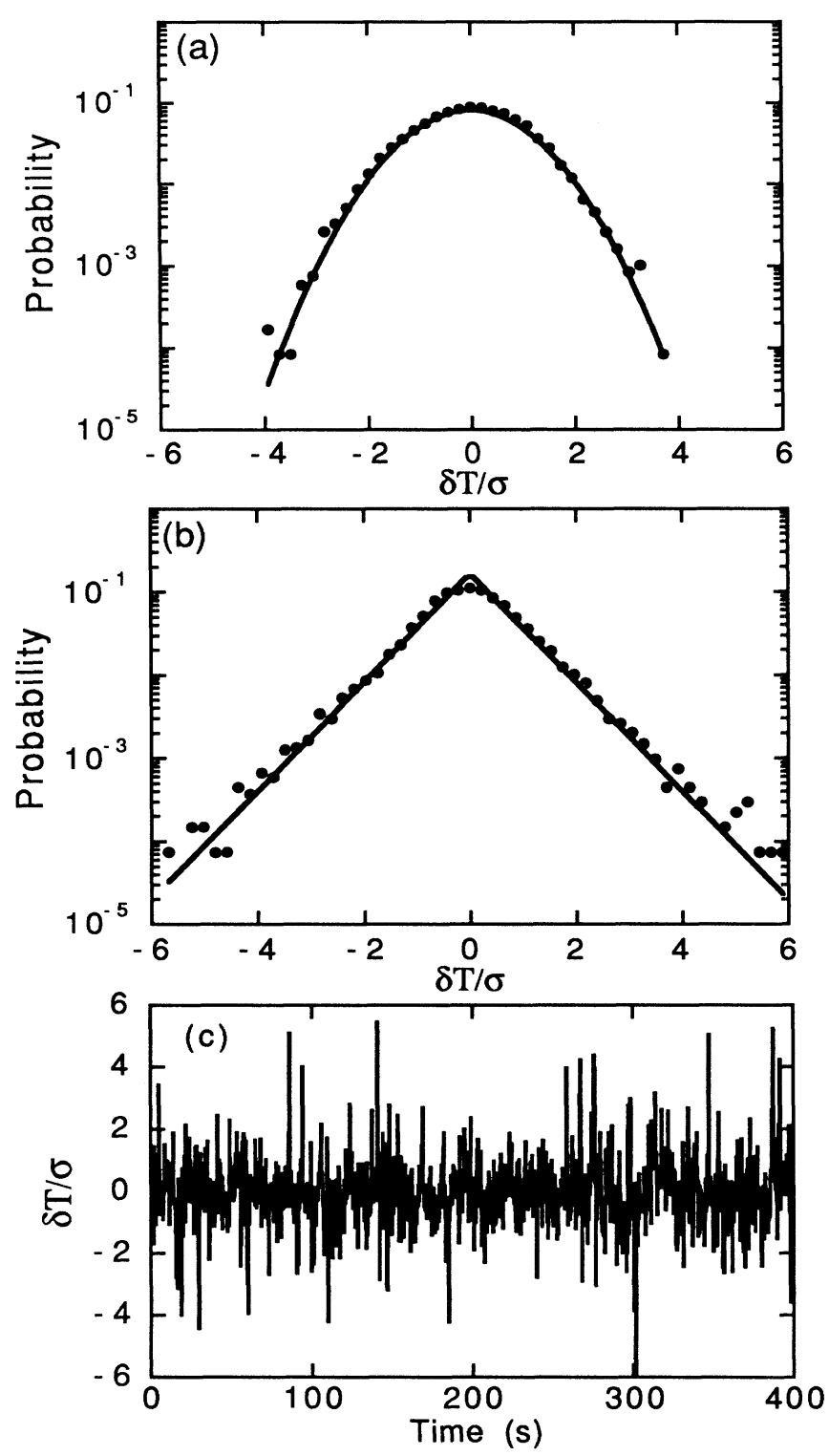

FIG. 4. Temperature distributions (a) at $R=600$, with a Gaussian fit, and (b) at $R=3700$, with an exponential fit. (c) A compressed time series corresponding to (b) showing frequent large fluctuations.

same variance as the data by a factor of 13 . A time series corresponding to Fig. 4(b), showing large fluctuations from the mean, is shown in Fig. 4(c).

The temperature gradient $\beta$ remains constant over a substantial distance (corresponding to at least $10 \sigma$ ), as shown in Fig. 5. The inverse decay constant $\gamma^{-1}$ of the exponential is predicted in Ref. [5] to be of order $\beta \xi_{V}$ $\left(=0.026 \mathrm{~K}\right.$, if we take $\left.\xi_{V}=d\right)$. The observed value of $0.029 \mathrm{~K}$ is consistent with this prediction.

To characterize the extent of non-Gaussian behavior as a function of $R$, we measured the kurtosis $\left\langle(\delta T)^{4}\right\rangle /$ $\left\langle(\delta T)^{2}\right\rangle^{2}$, which is 3 for a Gaussian distribution and 6 for

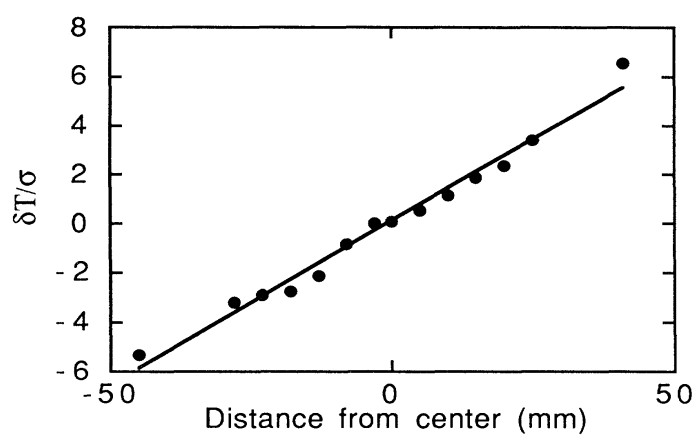

FIG. 5. Mean temperature profile for $R=3700$, showing linearity over a distance corresponding to at least $10 \sigma$, where $\sigma=0.042 \mathrm{~K}$ is the standard deviation of the distribution of Fig. 4(b).

an exponential distribution extending to the center. The observed values, shown in Fig. 6, are consistent with 3 at low $R$, and rise steadily to the range 5-6 for $R \approx 4000$. The scatter in the kurtosis is somewhat larger than one might expect. The distributions are also sometimes skewed by as much as $10 \%$, and the degree of rounding at the top of the distribution varies. These variations may arise from slight drifts in the temperature control or environment during the runs, but are not sufficiently serious to mask the clear trend toward exponential distributions above the mixing transition.

We confirmed by using glycerol-water mixtures that the distributions do not vary significantly for a factor of 5 increase in Prandtl number $v / \kappa$, where $v$ is the kinematic viscosity and $\kappa$ the thermal diffusivity. (However, only the range $R<1000$ was accessible in that case.) Finally, the distributions were approximately Gaussian when $\xi_{V} / L$ was increased to 0.15 by changing the grid, up to $R$ $=16000$.

Additional experiments were conducted in a different geometry, in which disordered capillary waves induced by the Faraday instability produced the mixing instead of an oscillating grid. This flow was harder to characterize in detail, but some evidence for exponential distributions

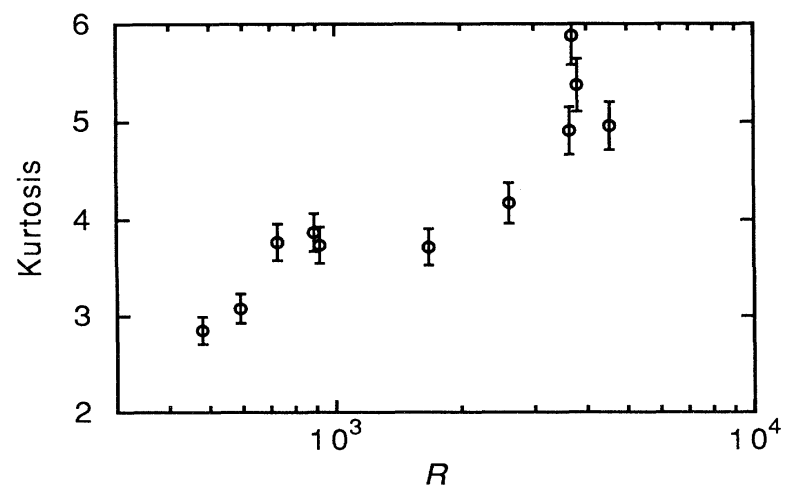

FIG. 6. Kurtosis as a function of $R$. This quantity would be 3 for Gaussian distributions and 6 for exponentials. 
was also found in this case for sufficiently strong mixing.

In summary, we have studied both the effective or "eddy" diffusivity and the statistics of local fluctuations of a passive scalar in the presence of a linear mean profile. We found clear evidence for exponential tails in the probability distributions for strong mixing, as suggested by Pumir, Shraiman, and Siggia [5], and Gaussian behavior for weaker mixing. The fact that the exponentials can extend nearly to the center of the distribution, as indicated by values of the kurtosis approaching 6, was not anticipated theoretically. We note that the mixing in our experiment was always too strong to produce nonmonotonic distributions with spikes at the boundary temperatures, but bimodal distributions have been seen in mixing layers [11].

The extent to which the statistics of the velocity fluctuations or the anisotropy of the velocity field might affect the scalar distributions remains to be established. It would also be of interest to extend these measurements to higher Reynolds numbers.

The authors thank B. Shraiman and E. Siggia for suggesting this experiment and for helpful discussions. We also appreciate discussions with R. Kraichnan and Z.-S. She. S. Gaudio built the apparatus. The research was supported by DARPA/URI under Contracts No. N00014-86-K-0758 and No. N00015-85-K-0759. O.N.M. acknowledges partial support from the Brazilian agency Conselho Nacional de Desenvolvimento Cientifico e Tecnologico.

(a) To whom correspondence should be addressed. Electronic address: jgollub@acc.haverford.edu.

(b) Permanent address: Departamento de Fisica, Universidade Federal de Minas Gerais, 30.161 Belo Horizonte/MG, Brazil.

[1] B. Castaing et al., J. Fluid Mech. 204, I (1989); M. Sano, X.-Z. Wu, and A. Libchaber, Phys. Rev. A 40, 6421 (1989); T. H. Solomon and J. P. Gollub, Phys. Rev. A 43, 6683 (1991).

[2] D. P. Lathrop, J. Fineberg, and H. L. Swinney (to be published).

[3] H. Aref, Phys. Fluids A 3, 1009 (1991), and other articles in the same volume.

[4] C. W. van Atta and W. Y. Chen, J. Fluid Mech. 44, 145 (1970); R. H. Kraichnan, Phys. Rev. Lett. 65, 575 (1990); Z.-S. She, Phys. Rev. Lett. 66, 600 (1991); Z.-S. She and S. A. Orszag, Phys. Rev. Lett. 66, 1701 (1991); R. Antonia, E. Hopfinger, Y. Gagne, and F. Anselmet, Phys. Rev. A 30, 2704 (1984); B. Castaing, Y. Gagne, and E. J. Hopfinger, Physica (Amsterdam) 46D, 177 (1990).

[5] A. Pumir, B. Shraiman, and E. D. Siggia, Phys. Rev. Lett. 66, 2984 (1991).

[6] O. Metais and M. Lesieur, in Advances in Turbulence II, edited by H.-H. Fernholz and H. E. Fielder (SpringerVerlag, Heidelberg, 1989), p. 371.

[7] E. D. Siggia (private communication); A. R. Kerstein, J. Fluid Mech. 231, 361 (1991).

[8] M. Avellaneda and A. Majda (to be published).

[9] Ya. G. Sinai and V. Yakhot, Phys. Rev. Lett. 63, 1962 (1989); H. Chen, S. Chen, and R. H. Kraichnan, Phys. Rev. Lett. 63, 2657 (1989).

[10] R. H. Kraichnan (private communication).

[11] M. M. Koochesfahani and P. E. Dimotakis, J. Fluid. Mech. 170, 83 (1986). 